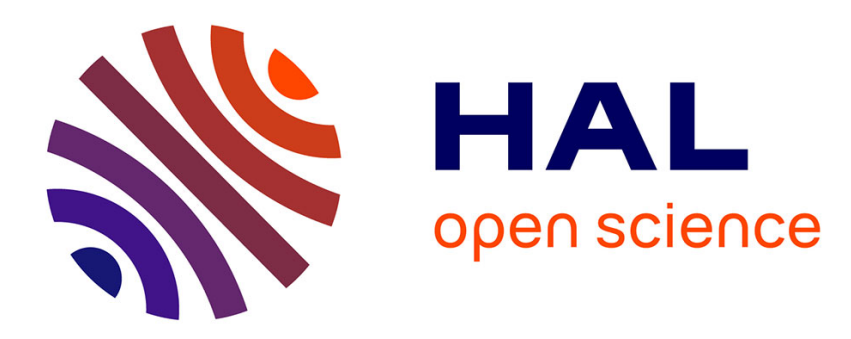

\title{
Les titulatures d'oratoires dans le département du Var Joël Candau
}

\section{To cite this version:}

Joël Candau. Les titulatures d'oratoires dans le département du Var. Le monde alpin et rhodanien, 1990. halshs-01723724

\section{HAL Id: halshs-01723724 \\ https://shs.hal.science/halshs-01723724}

Submitted on 5 Mar 2018

HAL is a multi-disciplinary open access archive for the deposit and dissemination of scientific research documents, whether they are published or not. The documents may come from teaching and research institutions in France or abroad, or from public or private research centers.
L'archive ouverte pluridisciplinaire HAL, est destinée au dépôt et à la diffusion de documents scientifiques de niveau recherche, publiés ou non, émanant des établissements d'enseignement et de recherche français ou étrangers, des laboratoires publics ou privés. 


\section{Les titulatures d'oratoires dans le département du Var} Joël Candau

\section{Abstract}

Oratory titles in the Var department.

The ranking of favorite saints of the Var - determined according to the patron saints of the oratories in the department reveals something of the regional cultural identity : the Virgin Mary is first, followed in variable order by John the Baptist, Joseph, Magdalene, Ann, Peter, and Mark.

Adding dates to this corpus enriches the rank observations, and modifies somewhat their analysis. Comparing the oratories erected before and after 1955, we note an increase in favor of the Virgin, accompanied by a decrease in the pantheon of intercessors. However, this decrease is only apparent, since the oratories recently dedicated to Mary show the intention of the founders to make Mother of Christ their Our Lady, by the great variety of the personalized titles of the oratories.

The rise of the cuit of the Virgin thus masks the continuity of a devotion marked by the diversity of intercessors. But beyond this continuity, the recent titles also reveal a personalization and a secularization of the monuments. Recent names have a frankly private intent, and the functionalism and estheticism of the new edifices suggest an alteration of the founders' religious motivations.

This last observation opens onto the more general question of relations between cuit of saints and quest for identity. Whether old or new, oratory names operate as identity markers, but on différent levels - collective or private - without it being possible to dissociate them entirely.

\section{Citer ce document / Cite this document :}

Candau Joël. Les titulatures d'oratoires dans le département du Var. In: Le Monde alpin et rhodanien : revue régionale d'ethnologie, n¹-2/1990. pp. 65-84;

http://www.persee.fr/doc/mar_0758-4431_1990_num_18_1_1435 


\title{
Les titulatures d'oratoires dans le département du Var
}

\author{
Joël Candau
}

I Sol.é au bord d'un chemin, dressé dans un bois ou près d'un champ, souvent éloigné du bourg, doté d'une architecture généralement fruste, l'oratoire a toutes les apparences d'un monument anodin dont les dimensions modestes n'attirent pas l'attention. Sa profusion dans le sud-est de la France et, notamment, dans le $\mathrm{Var}^{(1)}$, en fait pourtant un objet ethnographique remarquable, ne serait-ce que parce qu'il permet de mieux cerner un domaine capital de la religion dite populaire : le culte des saints.

La recherche dont nous rendons compte ici a permis de rassembler deux types de données. Les premières, recueillies de manière systématique dans les inventaires et les archives des Amis des oratoires ${ }^{(2)}$, se rapportent à l'ensemble du département. Les secondes constituent un matériau ethnographique plus élaboré, obtenu grâce à l'enquête de terrain dans deux cantons du Var, le Beausset et Rians ${ }^{(3)}$, choisis pour leur richesse en monuments. Les titulatures d'oratoires évoquées dans cet article ne constituent qu'un volet particulier de cette recherche ${ }^{(4)}$.

(1) Sur les 6851 édicules recensés à la fin de l'année 1983 à l'intérieur du territoire français, 3868 (soit $56 \%$ ) sont regroupés dans huit départements du sud-est : Alpes-Maritimes, Alpes-de-Haute-Provence, Bouches-du-Rhône, Hautes-Alpes, Haute-Savoie, Savoie, Var, Vaucluse. Parmi eux, le Var est le mieux doté avec 838 monuments.

(2) Amis des oratoires, Maison des Agriculteurs, 22, av. Henri-Pontier, 13626, Aix-enProvence.

(3) Situés respectivement aux confins sud-ouest et nord-ouest du département du Var, les cantons du Beausset et de Rians sont inégalement pourvus en oratoires. En 1984, cing des six communes du canton du Beausset enfermaient 131 édicules : 50 au Beausset, 26 à Saint-Cyr, 25 au Castellet, 19 à La Cadière, 11 à Signes. Dans le canton de Rians, 46 monuments s'élevaient à la même date : 19 à Rians, 13 à Saint-Julien-Le-Montagnier, 8 à La Verdière, 4 à Artigues et 2 à Ginasservis. La commune de Riboux dans le canton du Beausset et celle de Vinon-sur-Verdon dans celui de Rians ne possèdent aucun oratoire.

(4) L'oratoire est un objet d'étude qui se prête en fait à de multiples exploitations. Si nous ne développons ici que la question des titulatures, nous nous permettons de renvoyer le

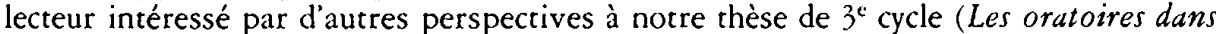
l'espace rural varois, Université de Nice - UER Lettres et Sciences humaines, juillet 1984) ainsi qu'à l'article « Les oratoires dans l'espace rural varois », Le Monde alpin et rbodanien, $\mathrm{n}^{\circ}$ 2-3/1985, pp. 83-107 avec bibliographie). 


\section{LE PALMARÈS}

Acte essentiellement individuel, la fondation d'un oratoire a pour dessein originaire la célébration d'un intercesseur particulier. L'ensemble des titulatures d'oratoires d'une région donnée rassemble de ce fait un véritable panthéon de saints thaumaturges dont on peut s'exercer à dresser le palmarès ${ }^{(5)}$.

Dans le Var, 116 saints différents se partagent 767 dédicaces connues ${ }^{(6)}$ des 838 oratoires recensés à l'époque de notre recherche ${ }^{(7)}$. La Vierge affirme sa suprématie, avec 261 citations, suivie de Saint Joseph (49), saint Jean-Baptiste (35), le Christ (27), saint Pierre (25), sainte MarieMagdeleine (24), sainte Anne (23), saint Antoine de Padoue (18), saint Eloi (16), saint Michel et saint Louis (13), saint Roch (12) saint Marc (11), saint Etienne (10), sainte Thérèse de l'Enfant Jésus (9), saint François d'Assise et saint Pons (8), saint Clair et saint André (7), quatre saints nommés six fois, quatre invoqués cinq fois, quatre autres cités quatre fois, seize saints mentionnés trois fois, treize intercesseurs bénéficiant de deux titulatures et cinquante-sept d'une seule. Les saints représentent $52 \%$ des vocables et les saintes $48 \%$.

Les 131 oratoires du canton du Beausset, riches de 126 titulatures, sont placés sous le patronage de 44 saints différents, 17 dédicaces restant indéterminées. Comme pour l'ensemble du département, le chiffre total des vocables connus et inconnus $(126+17)$ dépasse celui des édicules, certains oratoires honorant deux et parfois trois titulaires distincts.

La première place échoit de nouveau à la Vierge (38 titulatures). Puis viennent saint Jean-Baptiste (8), saint Joseph (6), sainte Anne, saint Antoine de Padoue, saint Etienne, sainte Magdeleine, saint Michel, saint François d'Assise et le Christ (4 dédicaces). Saint Roch, saint Louis et saint Eloi sont cités trois fois. Six autres saints (sainte Bernadette, saint Clair, saint Marc, saint Pierre, saint Philippe et saint Sébastien) apparaissent deux fois, et vingt-cinq une seule fois ${ }^{(8)}$. La part des saints dans les titulatures est de $57 \%$ contre $43 \%$ pour les saintes.

Nous ignorons la titulature de quatre édicules rianssais. Les 42 autres monuments, dotés de 45 vocables, sont placés sous l'invocation de 28 saints différents. Par rapport au Beausset, le déséquilibre entre les sexes s'aggrave, peut-être révélateur, dans cette région, d'un « tropisme cultuel

(5) Les vocables pris en compte ici sont des titulatures officielles, mais on a pu observer des monuments placés sous le patronage d'un seul intercesseur abritant en réalité dans leur niche deux statuettes de saints distincts. De même, en plus du titulaire principal, l'oratoire enferme parfois dans son pilier plusieurs médailles de saints secondaires.

(6) La titulature de 85 monuments reste inconnue.

(7) Recension de M. Louis JANVIl: (Inventaire des oratoires du Var, Aix-en-Provence, Amis des oratoires, 1982,121 p.), actualisée en janvier 1984 à partir des archives de l'association des Amis de l'oratoire et corrigée en fonction de notre propre inventaire dans les cantons du Beausset et de Rians.

(8) Alban, André, Barbe, Cécile, Charles Borromée, Côme, Damien, Daniel, Dominique, Elisabeth, Eutrope, François de Sales, Isidore, Jules, Laurent, Lucien, Manu, Marcel, Paul, Pierre és-liens, Rose, Sylvie, Thérèse, Victor, Yves. 


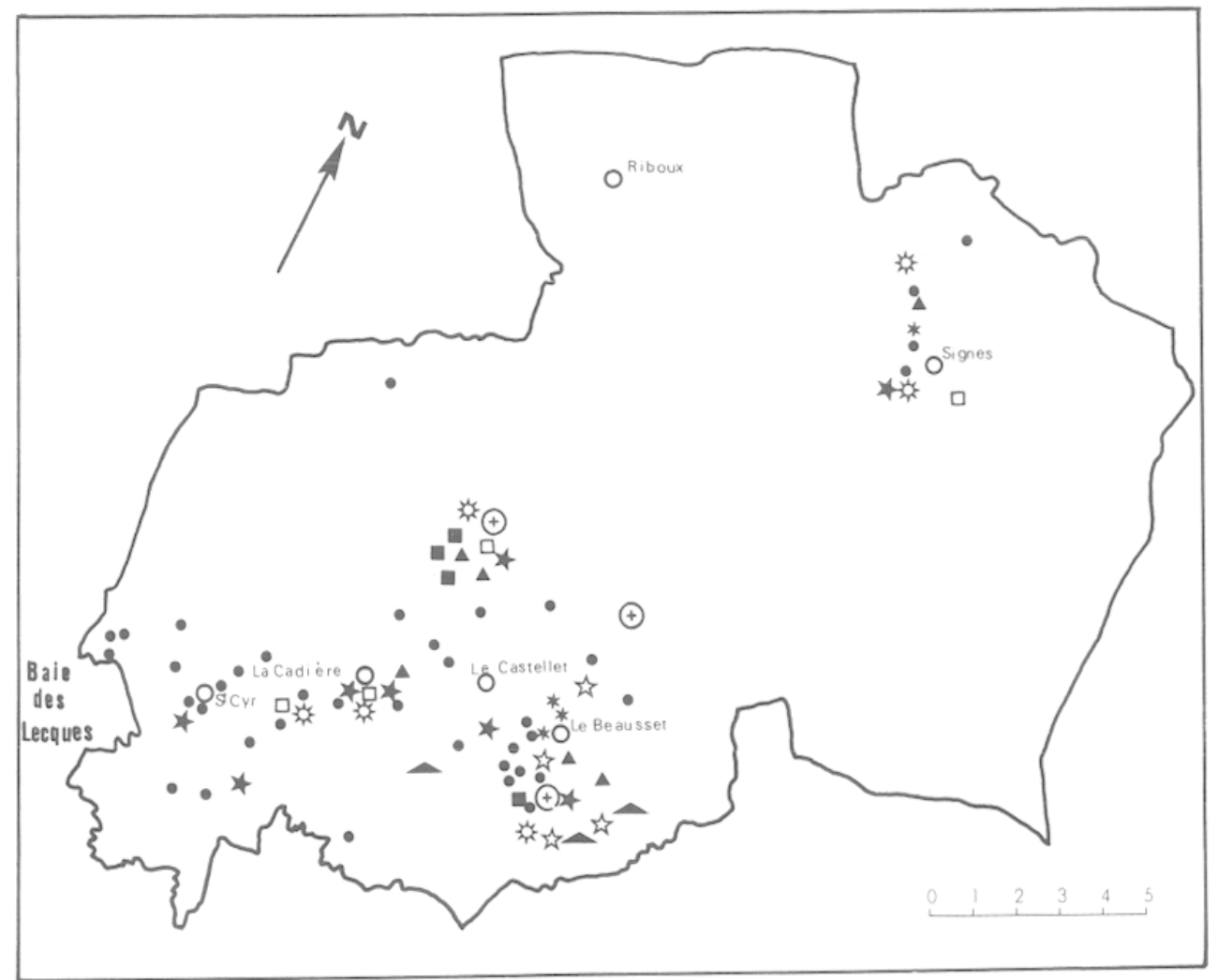

- Vierge

$\star \quad$ Sairt Jean-Baptiste

- Sairt Joseph

- Sairite Anne

* Sainte Marie-Magdeleine

- Saint Michel

4. Saint François d'Assise

( Saint Roch

- Saint Eloi

* Chapelles rurales

- Village

1. Les principaux titulaires du canton du Beausset.

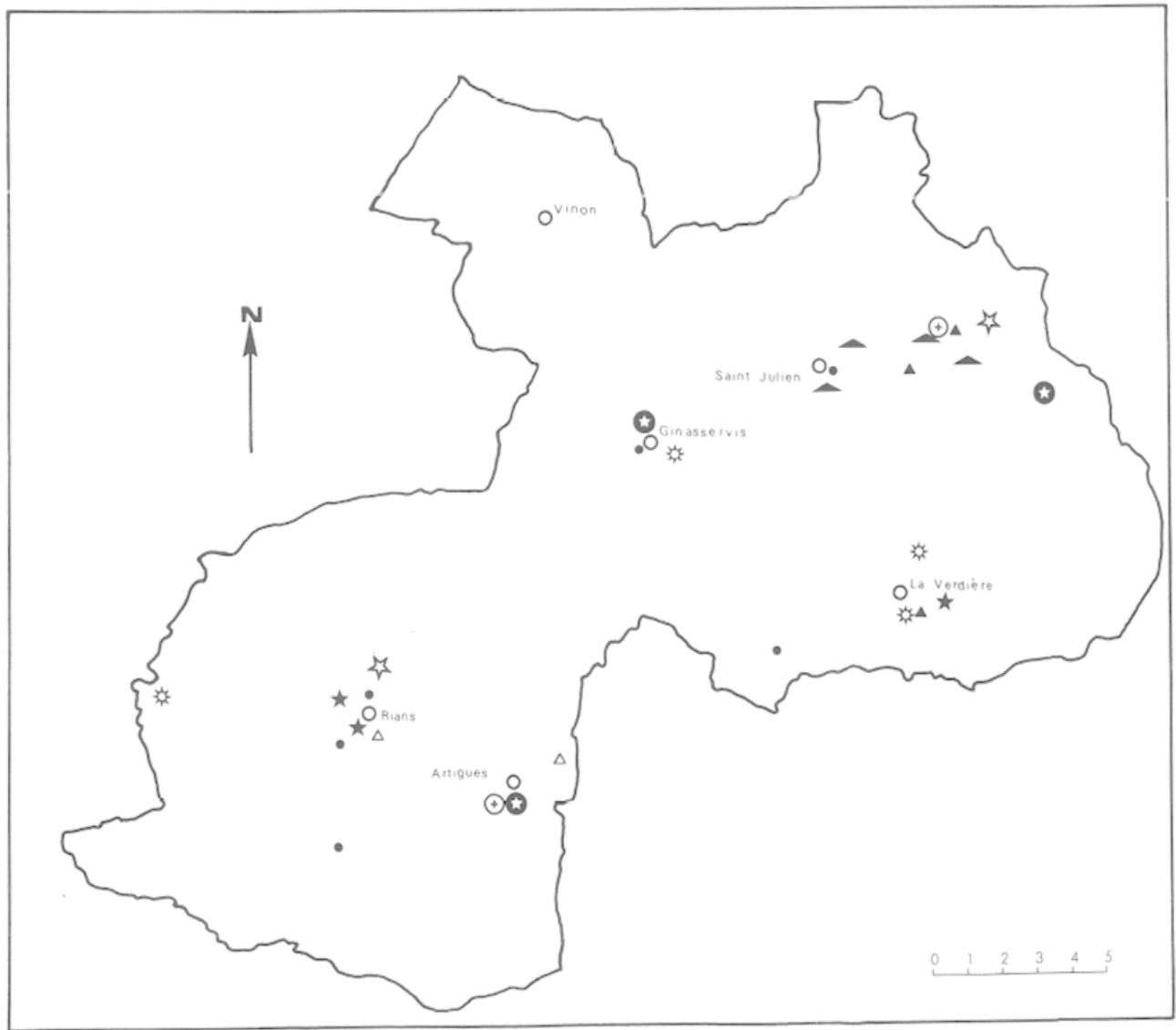

- Vierge

- Saint Eloi

$\star \quad$ Saint Jean-Baptiste

- Saint Joseph

* Saint Pierre

$\triangle$ Saint Honorat

kै Saint Marc

$\oplus$ Soint Roch

Chapelles rurales

o Village

2. Les principaux titulaires du canton de Rians. 


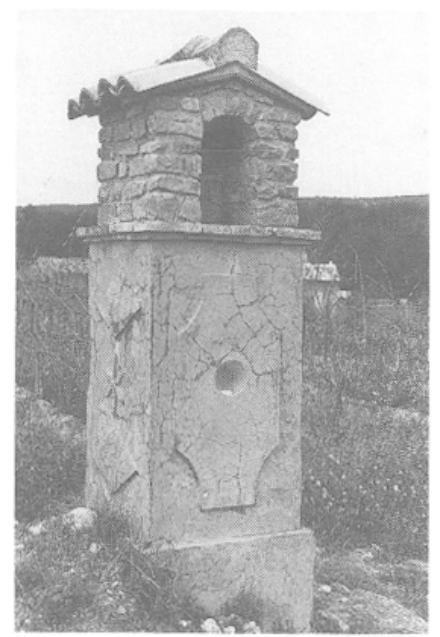

Saint Jean-Baptiste, Le Castellet. Oratoire ancien. Localisation : ager, bord d'un chemin. Orientation: $160^{\circ}$. En pierres crépies, niche cintrée en pierres sur corniche. Toit en bâtière en tuiles creuses. Bénitier. Moulures sur le devant et les côtés. Pas de statuette. Dimensions, $h: 229 \times 1: 67$ $x p: 67$. Niche, $h: 40 \times 1: 24 \times$ $p: 30$. mâle » ${ }^{(9)}$ : les saints (73\% des titulatures) l'emportent nettement sur les saintes $(27 \%)$.

La Vierge reste la première citée (six dédicaces), suivie par saint Eloi (quatre), saint Jean-Baptiste, saint Joseph et saint Pierre (trois), puis par saint Honorat, saint Marc et saint Roch (deux). Vingt intercesseurs n'ont qu'une seule titulature ${ }^{(10)}$.

Comme on peut le constater sur les cartes cantonales ( ${ }^{\text {os }} 1$ et 2 ), l'implantation des principaux saints est très inégale. Précédé par la Vierge, présente dans neuf communes sur les dix pourvues d'oratoires, saint Jean-Baptiste reste le "saint le plus populaire en Provence " (11), objet, toujours, d'une dévotion vivace comme en témoignent son occurrence dans sept communes différentes. Seul saint Joseph apparaît ensuite dans plus de la moitié des localités (dans six exactement), tous les autres titulaires restant en-deçà de ce seuil.

Avec quelques nuances, le classement varois se retrouve dans plusieurs régions méridionales. En Ardèche, $30 \%$ des oratoires ont la Vierge pour titulaire, suivie par les saints Joseph, Anne, Roch, Marc, Pierre, Magdeleine, Antoine, Etienne, Louis, Eloi ${ }^{(12)}$. Dans le Vaucluse, les NotreDame devancent saint Joseph, saint Marc, saint Gens, saint Roch et saint Michel(13). Dans les Bouches-du-Rhône, la Vierge arrive encore en tête ${ }^{(14)}$. Des recensements incomplets mais qui portent sur toute la Provence ${ }^{(15)}$ donnent un palmarès semblable : Notre-Dame, saint Joseph, sainte Anne, saint Marc, saint Jean-Baptiste. Partout, la présence du Christ se révèle très faible ${ }^{(16)}$.

(9) Alphonse DUPRONT in Marie-Hélène FROLSCHIE-CHOPARD, La religion populaire en Provence orientale au XVIIle siècle, Paris, Editions Beauchesne, 1980, p. 10.

(10) André, Antoine de Padoue, Benoît Labre, Cécile, Denis, François d'Assise, François de Sales, Foy, Hubert, Laurent, Louis de Gonzague, Magdeleine, Martin, Michel, Saint Nom de Jésus, Philomène, Roseline, Sébastien, Thérèse de l'Enfant Jésus, Vincent de Paul.

(11) Claude Seignolle, Le Folklore de la Provence, Paris, G.P. Maisonneuve et Larose, 1963 , p. 203. Rappelons que les départements avec plus de trois communes portant le nom de saint Jean se situent tous dans une zone géographique qui correspond à peu près à la moitié sud de la France. Voir Emmanuel I.l: ROY LADURIE, A. ZYSBERG, «Géographie des hagiotoponymes en France », Annales E.S.C., n 6, nov-déc. 1963, p. 1308.

(12) Louis BRUN, Oratoires de nos terroirs, Nîmes, Louis Brun, 1976, pp. 67-68.

(13) René PAUPLIN, Les oratoires du Vaucluse, Aix-en-Provence, Editions des Amis des oratoires, 1970.

(14) Pierre IRIGOIN, Les oratoires des Bouches-du-Rbône, Aix-en-Provence, Editions des Amis des oratoires, 1947.

(15) G. ARnaud DAGNil, L. DOR, Les oratoires de Provence, Cannes, Editions Delonnoy, 1938 , p.53.

(16) Au-delà des changements qui affectent l'ordre des saints à l'intérieur de ce peloton de tête, celui-ci dénote une relative fixité des vocables, observée ailleurs que dans les titulatures d'oratoires. Au XVIII ${ }^{\mathrm{e}}$ siècle, l'ordre était le suivant pour l'ensemble des saints honorés dans l'ancien diocèse d'Aix-en-Provence: la Vierge (un tiers des appellations) suivie de saint Pierre (en régression), saint Roch, sainte Anne et saint Joseph. Voir à ce sujet J. DI: FONT-RI:AULX, « Les saints honorés dans l'ancien diocèse d'Aix », Provence bistorique, juil.-sept. 1972, pp. 35-52. A la même époque, MarieHélène Froschlé-Chopard a mis en évidence un classement des cultes (églises, chapelles, prieurés, toponymie religieuse) très proche du nôtre, dans les diocèses de Vence-Grasse, Fréjus, Toulon, Aix, Marseille. Marie-Hélène FroESCHLE-CHOPARD, "L'espace et le sacré au XVIII ${ }^{\epsilon}$ siècle », Annales de Bretagne et des pays de l'Ouest ", tome $90,1983, \mathrm{n}^{\circ} 2$, p. 207. 
Les deux tableaux suivants résument les données varoises. La richesse du panthéon des intercesseurs apparaît nettement, en particulier à Rians où on relève 28 titulatures distinctes, soit $62 \%$ des dédicaces connues, pour 46 monuments seulement. Ce pourcentage diminue cependant lorsqu'on passe de Rians au Beausset, puis au département: il évolue, chaque fois de manière inversement proportionnelle au nombre de monuments et à celui des dédicaces. Tout se passe comme si, au moment de l'élection d'un titulaire, les autochtones disposaient d'un répertoire de noms de saints suffisamment vaste pour permettre un choix, mais néanmoins limité par rapport à l'éventail beaucoup plus large des noms possibles $^{(17)}$.

\begin{tabular}{|l|c|c|c|c|}
\hline $\begin{array}{c}\text { Canton } \\
\text { ou } \\
\text { département }\end{array}$ & $\begin{array}{c}\text { Nombre } \\
\text { de } \\
\text { monuments }\end{array}$ & $\begin{array}{c}\text { Dédicaces } \\
\text { connues }\end{array}$ & $\begin{array}{c}\text { Dédicaces } \\
\text { inconnues }\end{array}$ & $\begin{array}{c}\text { Nombre de titula- } \\
\text { tures distinctes et } \\
\% \text { de celles-ci par } \\
\text { rapport au nombre } \\
\text { total de dédicaces } \\
\text { connues }\end{array}$ \\
\hline Le Beausset & 131 & 126 & 17 & $44 \quad(35 \%)$ \\
Rians & 46 & 45 & 4 & $28 \quad(62 \%)$ \\
Var & 838 & 767 & 85 & $116 \quad(15 \%)$ \\
\hline
\end{tabular}

3. Nombre de dédicaces connues, inconnues et de titulatures distinctes.

\begin{tabular}{|c|c|c|c|c|c|c|}
\hline $\begin{array}{c}\text { Canton } \\
\text { ou } \\
\text { département }\end{array}$ & & Saints & & Saintes & $\begin{array}{l}\text { Part prise par } \\
\text { la Vierge dans } \\
\text { la catégorie } \\
\text { des saintes }\end{array}$ & $\begin{array}{l}\text { Part prise par la } \\
\text { Vierge dans le total } \\
\text { des dédicaces } \\
\text { connues }\end{array}$ \\
\hline Le Beausset & 72 & $(57 \%)$ & & $(43 \%)$ & $38 \quad(70 \%)$ & $30 \%$ \\
\hline Rians & 33 & $(73 \%)$ & 12 & $(27 \%)$ & $6 \quad(50 \%)$ & $13 \%$ \\
\hline Var & 399 & $(52 \%)$ & 368 & $(48 \%)$ & $261 \quad(71 \%)$ & $34 \%$ \\
\hline
\end{tabular}

4. La Vierge, les saints et les saintes.

Il n'y a là qu'un constat très banal: les habitants de chaque région française manifestent généralement leur préférence pour certains saints qu'ils s'approprient et qui participent peu ou prou de ce qu'il est permis d'appeler l'identité cultuelle régionale. Une augmentation du nombre des édicules aboutit, à partir d'un certain seuil, à une clôture de ce fonds hagiographique local dans lequel puisent les fondateurs d'oratoires, avec pour corollaire la répétition de vocables déjà utilisés et, subséquemment, la diminution de la part relative des titulatures distinctes.

Ce phénomène semble profiter principalement à la Vierge, le nombre des titulatures mariales croissant en même temps que celui des

(17) Les 6851 édicules recensés en France par les Amis des oratoires et répertoriés dans leur fichier ont pour titulaires 351 saints différents. La Vierge arrive largement en tête avec 1650 vocables, suivie de saint Joseph, sainte Anne, saint Antoine et saint Roch. Les dédicaces de près d'un millier d'oratoires sont inconnues. 


\section{PÉRIODISATION}

La périodisation des données enrichit ces premières observations et modifie partiellement leur analyse ${ }^{(18)}$. Abandonnons le Var dans son ensemble, la datation des monuments souffrant de trop de lacunes dans tous les cantons où il n'y a pas eu d'enquête de terrain, afin d'examiner de plus près les données relatives aux oratoires des deux cantons réunis ${ }^{(19)}$ (tableau $n^{\circ}$ 6).

\begin{tabular}{|c|c|c|c|c|c|}
\hline Cantons & $\begin{array}{c}\text { Périodisation } \\
\text { des } \\
\text { oratoires }\end{array}$ & $\begin{array}{c}\text { Nombre } \\
\text { de } \\
\text { monuments }\end{array}$ & $\begin{array}{c}\text { Dédicaces } \\
\text { connues }\end{array}$ & $\begin{array}{c}\text { Dédicaces } \\
\text { inconnues }\end{array}$ & $\begin{array}{c}\text { Nombre de titula- } \\
\text { tures distinctes et } \\
\text { part prise par celles- } \\
\text { ci dans le nombre } \\
\text { total de dédicaces } \\
\text { connues }\end{array}$ \\
\hline $\begin{array}{l}\text { Total } \\
\text { Le Beausset } \\
+ \text { Rians) }\end{array}$ & anciens & 112 & 107 & 14 & $44 \quad(41 \%)$ \\
récents & 65 & 64 & 7 & $27 \quad(42 \%)$ \\
\hline
\end{tabular}

6. Périodisation du nombre de dédicaces connues et inconnues et des titulatures distinctes.

La diversité des titulatures des monuments anciens est plus forte que celle des édicules récents en valeur absolue (44 contre 27) mais sensiblement égale en valeur relative ( $41 \%$ contre $42 \%$ ). Le phénomène de clôture du registre hagiographique local semble par conséquent jouer dans la catégorie des monuments anciens, gratifiés d'un nombre de titulatures connues nettcment plus grand que le groupe des oratoires récents (107 contre 64). Dans celui-ci, ce processus se manifeste beaucoup plus faiblement, hypothèse étayée par le fait que $7 \%$ seulement des titulatures distinctes d'oratoires récents sont communes aux deux cantons, contre $26 \%$ dans le cas des oratoires anciens.

Cependant, un examen plus attentif des données laisse penser qu'un élargissement du corpus altèrerait davantage la diversité des titulatures dans le groupe des oratoires récents que dans celui des monuments anciens. En effet, dans le premier groupe, le graphique $n^{\circ} 7$ montre le choix d'un nombre plus réduit d'intercesseurs distincts dans la classe des saints majoritaires : alors qu'il faut huit saints différents pour regrouper la

(18) Confronté à d'importants problèmes de datation des monuments les plus anciens, nous avons opté pour une périodisation minimum, en nous limitant à distinguer au sein de notre corpus les oratoires récents et les édicules anciens. Le milieu des années 1950 fut choisi comme seuil de bipartition. A cette époque, tout un ensemble d'indicateurs (baissc dc la population active agricole, essor démographique dû à un apport extérieur, début des ventes massives de terres agricoles au profit de la fonction résidentielle, augmentation du taux moyen d'abandon annuel des cultures, progression fulgurante des demandes de permis de construire) laissaient deviner une accélération des mutations qui, dans la région, transformaient des communautés encore majoritairement paysannes. Sommaire, sans aucun doute (la catégorie des oratoires anciens rassemble des édicules érigés entre le XVII ${ }^{\mathrm{e}}$ siècle et 1955 ), mais imposée par la nature même des sources, cette mise en contexte historique s'est révélée pertinente et opératoire tout au long de la recherche.

(19) Du fait de ce petit nombre d'oratoires récents à Rians (cinq) nous avons renoncé à distinguer les deux cantons lors de chaque périodisation des données. 
moitié des dédicaces des oratoires anciens, ce seuil est atteint dès le troisième titulaire avec les oratoires récents. Du point de vue du nombre des édicules, on note ici par conséquent une évolution inverse de celle observée dans le graphique $n^{\circ} 4$ puisque, dans le cas présent, le seuil de $50 \%$ est atteint plus rapidement avec les oratoires récents, bien que leur nombre (65) soit très inférieur à celui des édicules anciens (112).
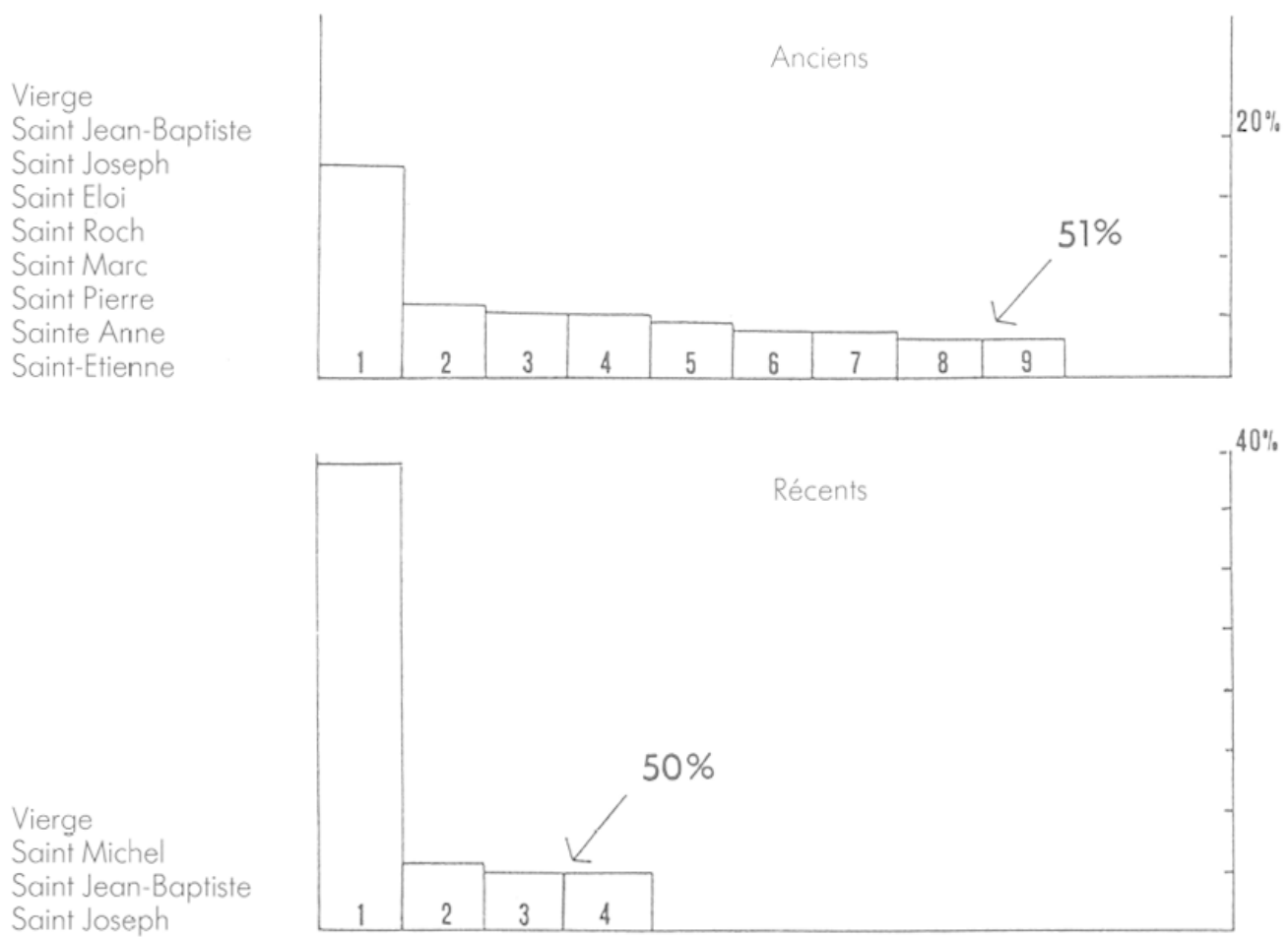

6. Le réemploi des saints les plus cités: périodisation.

Cette constitution plus rapide d'un groupe de saints majoritaires a pour origine, essentiellement, l'augmentation du nombre de vocables NotreDame (tableau $n^{\circ} 8$ ). Leur part croît dans la catégorie des saintes (de $56 \%$ à $78 \%$ ) comme par rapport au total des dédicaces connues où elle fait plus que doubler (de $18 \%$ à $39 \%$ ), ce qui explique ici encore l'émergence d'un équilibre entre les titulatures masculines et féminines $(50 \%$ de part et d'autre).

\begin{tabular}{|c|c|c|c|c|c|}
\hline Cantons & $\begin{array}{c}\text { Périodisation } \\
\text { des } \\
\text { oratoires }\end{array}$ & Saints & Saintes & $\begin{array}{c}\text { Part prise par la } \\
\text { Vierge dans la } \\
\text { catégorie des } \\
\text { saintes }\end{array}$ & $\begin{array}{c}\text { Part prise par la } \\
\text { Vierge dans le } \\
\text { total des dédi- } \\
\text { caces connues }\end{array}$ \\
\hline $\begin{array}{l}\text { Total } \\
\text { (Le Beausset } \\
+ \text { Rians) }\end{array}$ & anciens & $73 \quad(68 \%)$ & $34 \quad(32 \%)$ & $19 \quad(56 \%)$ & $18 \%$ \\
\hline
\end{tabular}

8. Périodisation: la Vierge, les saints et les saintes. 
Retenons ce double mouvement lors du passage des oratoires anciens aux édicules récents : la progression de la Vierge et ce qu'on peut appeler l'appauvrissement du panthéon, caractérisé par la répétition d'une partie des titulatures aux dépens des autres possibles. Ce dernier phénomène ne peut résulter uniquement d'une augmentation du corpus des monuments comme nous le supposions au début puisque, on vient de le constater, ce processus se manifeste pareillement dans la catégorie des oratoires récents, pourtant moins nombreux que les monuments anciens. On peut donc présumer l'existence d'un autre facteur que la simple clôture du répertoire local des noms de saints, élément qui doit contribuer lui aussi à l'étiolement de la diversité de la Cour céleste. Quel est-il ? On croit déceler une partie de la réponse dans la progression de la Vierge.

\section{LES TITULATURES NOTRE-DAME}

La suprématie de la Vierge était attendue dans cette région de forte dévotion mariale qu'est la Provence. Observée dans les fêtes ${ }^{(20)}$, les titulatures de sanctuaires ${ }^{(21)}$ ou dans la hiérarchie des intercesseurs représentés sur les ex-voto imagés ${ }^{(22)}$, cette précellence de la Vierge trouve au moins deux explications : l'attitude du clergé d'abord, en général beaucoup mieux disposé à l'égard du culte marial qu'envers celui des saints titulaires $^{(23)}$; la polyvalence de la Vierge, en second lieu, contrairement aux autres saints plus ou moins spécialisés dans un registre thaumaturgique singulier. Sous les regards de l'avocate la plus écoutée ${ }^{(24)}$, de la Grande Consolatrice :

(20) Dans les fêtes provençales des XVIII" et XIX" siècles, on obtient l'ordre suivant : fêtes mariales, fêtes de saint Jean-Baptiste, de saint Eloi et de saint Pierre. Michel Vovil.LE, Les métamorphoses de la fête en Provence de 1750 à 1820, Paris, Aubier-Flammarion, 1976 , p. 35.

(21) Près de $45 \%$ des chapelles de pèlerinage ce l'ancienne Provence sont sous l'invocation de la Vierge. Agnes EIGLIF:HAYOT, Pèlerinages et pèlerins dans l'ancienne Provence, Aix-en-Provence, thèse de $3^{\mathrm{e}}$ cycle, 1978, p. 16.

(22) Les ex-voto sont majoritairement adressés à la Vierge, suivie de loin par saint JeanBapriste, sainte Anne et le Christ. Bernard Cousin, Le miracle et le quotidien. Les exvoto provençux, image d'une société, Lniversité de Provence, Thèse de Doctorat d'Etat, 1981, p. 344.

(23) Le pourchas, par le clergé, des "saints inquiétants quant à leur réalité historique » (Alphonse I)t.PRONT, op. cit., p. 8) a pu inciter les fidèles à se tourner vers des intercesseurs moins conflictuels, quil s'agisse de saints liturgiques ou de la Vierge. Dans plusieurs communes des deux cantons étudiés, nous avons pu noter de telles substitutions de titulatures, la Vierge, saint Pierre ou saint Paul remplaçant par exemple les saints Casimir, Hippolyte ou Eloi. On doit néanmoins apprécier la pression cléricale avec beaucoup de nuances: dans l'Inventaire des oratoires du Var de $\mathbf{M}$. Louis JANVIER (op. cit.), nous avons compté 127 consécrations d'oratoires anciens et récents, aux titulatures les plus diverses, consécrations pratiquées principalement par le curé de la paroisse mais aussi, parfois (dans $15 \%$ des cas relevés), par un membre plus élevé de la hiérarchie ecclésiastique (évêque, archevêque).

(24) Les armoiries de la commune du Beausset représentent une Vierge d'or sous la devise : «Beaussentensium Advocata » (Avocate du Beausset). 


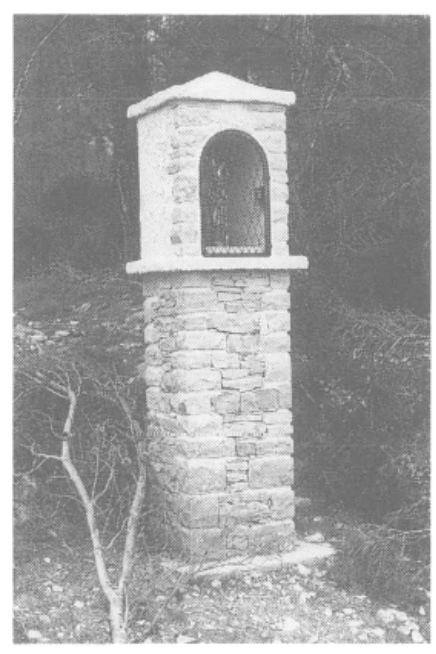

Notre-Dame des Restanques, Le Castellet. Oratoire récent. Localisation : saltus, bord d'un chemin. Orientation: $230^{\circ}$. En pierres taillées sur un petit socle en ciment. Niche crépie sur corniche avec montants en piverres. Grille en fer, statuette dorée représentant la Vierge à l'Enfant (h: 40). Inscription dans la niche, sur fond doré : "Notre dame des Restanques $»$. Dimensions $: h: 242 \times 1:$ $60 \times p: 60$. Niche $: h: 60 \times 1: 38$ $\times p: 49$.
«Les maladies guérissent, les fléaux disparaissent, les tempêtes, les orages violents s'apaisent, les naufrages sont évités ${ }^{(25)}$, les troubles sont dissipés. » $(26)$

Tous, clercs et laïcs, partagent cette idée d'un pouvoir très étendu de la Vierge :

« Si tous les saints peuvent intercéder pour nous et si Dieu daigne écouter leurs prières, à plus forte raison la Sainte Vierge, plus favorisée de Dieu, plus riche en mérites et élevée à un plus haut degré de gloire que tous les autres saints, a un pouvoir d'intercession, et est digne de nos hommages, de notre dévotion, de notre confiance. »(27)

Sa qualité de mère du Christ confère à la Vierge une dimension sotériologique absente chez les saints titulaires: ses liens maternels avec le Christ lui assurent une écoute plus attentive de celui-ci que celle dont bénéficient les autres intercesseurs. "La famille a toujours été attachée à sainte Cécile, mais la Mère de Dieu c'est encore mieux que les saints", déclare aujourd'hui le propriétaire d'un oratoire sis à Saint-Cyr pour expliquer le remplacement de sainte Cécile par Notre-Dame-des-Roses. Si cette dernière est "mieux que les saints", si les fidèles la perçoivent comme détentrice d'un «surcroît de spiritualité » ${ }^{(28)}$, alors comprend-on plus aisément une «mariolâtrie » ${ }^{(29)}$ grandissante et l'appauvrissement du panthéon des titulaires au profit de la Vierge ${ }^{(30)}$.

L'universalité de cette "médiatrice par excellence »(31) ne doit néanmoins pas faire illusion. Il n'est pas assuré que, pour les fondateurs d'un oratoire en l'honneur d'une Notre-Dame, il s'agisse partout de la même. La variété des appellations de la Vierge suggère un tel point de vue. En lui donnant un vocable particulier, chaque fondateur d'oratoire peut faire de la Vierge sa Notre-Dame. Les dédicaces choisies sont souvent une manière tout à fait explicite de s'approprier localement la Mère de Dieu.

(25) Dans le canton du Beausset, le fondateur d'un oratoire récent dédié à la Vierge justifie la force de sa dévotion par le fait que son fils sortit un jour indemne d'une violente tempête en mer.

(26) Prière adressée à Notre-Dame de Santé de La Verdière, Lucien PHILIBERT, Monographie du sanctuaire de Notre-Dame de La Verdière, Draguignan, Imprimerie du Var, 1916, p. 76.

(27) Abbé P.M. Roux, Le Beausset. Etude historique et géographique, Marseille-Toulon, 1910 , P. 17.

(28) Jean ARrouYe, « La dénonciation. A propos d'un ex-voto d'Allauch, 1817 », Provence historique, juil.-août-sept. 1981, 31, 125, p. 218.

(29) Michel VOVELLE, "La religion populaire : problèmes et méthodes », Le Monde Alpin et Rbodanien, ${ }^{\circ} 1-4 / 1977$, p. 24.

(30) Il serait erroné de vouloir expliquer l'appauvrissement du panthéon par la seule faveur actielle des titulatures mariales. D'autres facteurs jouent probablement, et parmi ceuxci sans doute faudrait-il évoquer le recul d'une certaine culture hagiographique : le répertoire des noms de saints dont disposent les fondateurs d'oratoires - nous entendons par là une connaissance minimum des récits et légendes définissant une vie de saint - est, peut-être, beaucoup moins fourni aujourd'hui qu'il ne l'était il y a quelques décennies. Bien évidemment, seule une enquête approfondie permettrait d'éprouver une telle hypothèse.

(31) M.H. FROESCHLE-CHOPARD, La Religion populaire en Provence orientale au XVIII siècle, op. cit., p. 107. 
On trouve dans les oratoires varois aussi bien des appellations désignant un lieu particulier - Notre-Dame des Champs, des Cyprès, des Farigoulettes, des Vignes, des Restanques -, que des vocables qui rappellent un événement familial. A Saint-Cyr, le fondateur de l'oratoire Notre-Dame de la Maintenance lui a donné ce nom "pour glorifier le fait que notre famille se soit maintenue sur notre propriété depuis trois siècles ». Il existe d'autres vocables, tels que Notre-Dame du Bon Sens au Beausset ou Notre-Dame de l'Aïoli à Toulon, dont loriginalité semble témoigner de la volonté de chaque fondateur de faire sienne une forme de dévotion partagée par le plus grand nombre ${ }^{(32)}$.

La périodisation appliquée aux seuls vocables Notre-Dame montre qu'un tel phénomène devient plus accusé lorsqu'on passe des oratoires anciens aux édicules récents.

Les deux tableaux suivants ( $\mathrm{n}^{\text {os }} 9$ et 10 ) rassemblent plusieurs séries de données. Le premier présente le nombre total de titulatures mariales et celui des titulatures distinctes, celles-ci reprises dans les trois dernières colonnes où sont regroupées: les appellations génériques sans autre précision sur la nature de la titulaire; les titulatures Notre-Dame de Lourdes ; les autres dédicaces spécifiques (Notre-Dame des Roses, du Bon Sens, des Champs, etc.). Dans le second tableau, inspiré par des travaux de Jacques Chiffoleau ${ }^{(33)}$, nous avons distingué à l'aide des vocables et de la statuaire les Vierges pathétiques (Notre-Dame de Pitié, Notre-Dame de la Solitude) ${ }^{(34)}$ et les Vierges apaisantes (Notre-Dame de la Garde, du Bon Sens, du Bon Accueil, des Victoires, des Roses, des Anges, de la Maintenance, de l'Assomption). Sont incluses dans cette catégorie, les Vierges «joyeuses » c'est-à-dire, pour reprendre la terminologie de Jacques Chiffoleau, celles qui font référence à la Nativité(35).

La diversité des dédicaces des oratoires récents (48\%) dépasse celle des monuments anciens (32\%). En revanche, l'occurrence des titulatures génériques (vocable Notre-Dame sans autre précision) est plus grande dans ceux-ci $(53 \%)$ que dans ceux-là $(36 \%)$. Il faut par conséquent

(32) Outre les appellations classiques (Notre-Dame de Lourdes, de la Garde, de la Salette, des Victoires, de la Médaille Miraculeuse, du Rosaire, des Anges, du Mai, Immaculée Conception, Mater Admirabilis), on rencontre encore dans le Var 52 titulatures mariales différentes : Notre-Dame du Peuple, des Pêcheurs, des Adieux, de la Route, du Bon Voyage, de la Sainte Espérance, du Bon Secours, de la Protection, de la Délivrance, du Petit Bon Dieu, dei Rougacion, Auxiliatrice, etc.

(33) Jacques Chiffolfau, La Comptabilité de l'au-delà, Rome, Ecole Française de Rome, 1980 , p. 371.

(34) Absentes dans les oratoires varois, on pourrait ranger dans cette catégorie les titulatures suivantes, rencontrées dans d'autres régions: Notre-Dame de Miséricorde, d'Humilité, Mater Dolorosa.

(35) Dans ce cas, l'enquête de terrain aide parfois à lever l'imprécision inhérente aux appellations génériques Notre-Dame. L'observation des statuettes permet en effet de distinguer une Vierge seule d'une Vierge à l'Enfant. Présente dans près du tiers (30\%) des titulatures mariales, cette dernière reste néanmoins minoritaire, ce qui correspond aux données rassemblées par B. Cousin dans sa thèse sur les ex-voto: le Var occidental préfère la Vierge seule à la Vierge à l'Enfant. Bernard Cousin, op. cit., p. 349. 


\begin{tabular}{|c|c|c|c|c|c|}
\hline \multirow{2}{*}{$\begin{array}{c}\text { Périodisation } \\
\text { des } \\
\text { oratoires }\end{array}$} & \multirow{2}{*}{$\begin{array}{c}\text { Nombre total } \\
\text { de titulatures } \\
\text { N.-D. }\end{array}$} & \multirow{2}{*}{$\begin{array}{l}\text { Titulatures } \\
\text { distinctes * }\end{array}$} & \multirow{2}{*}{$\begin{array}{l}\text { Titulatures } \\
\text { génériques }\end{array}$} & \multicolumn{2}{|c|}{ Titulatures spécifiques } \\
\hline & & & & N.-D. Lourdes & Autres \\
\hline Anciens & 19 & $6 \quad(32 \%)$ & $10(53 \%)$ & $5(26 \%)$ & $4(21 \%)$ \\
\hline Récents & 25 & $12(48 \%)$ & $9 \quad(36 \%)$ & $4(16 \%)$ & $12(48 \%)$ \\
\hline Total & 44 & $16(36 \%)$ & $19(43 \%)$ & $9 \quad(21 \%)$ & $16(36 \%)$ \\
\hline
\end{tabular}

\begin{tabular}{|c|c|c|}
\hline $\begin{array}{l}\text { Périodisation } \\
\text { des oratoires }\end{array}$ & $\begin{array}{l}\text { Vierges rassurantes ou } \\
\text { « joyeuses» }\end{array}$ & Vierges "pathétiques» \\
\hline Anciens & $6 \quad(32 \%)$ & $1 \quad(5 \%)$ \\
\hline Récents & $17 \quad(68 \%)$ & $1 \quad(4 \%)$ \\
\hline Total & $23 \quad(52 \%)$ & $2 \quad(4,5 \%)$ \\
\hline
\end{tabular}

* Dans cette colonne, et contrairement aux 4 autres colonnes, l'occurrence d'une titulature dans l'ensemble du corpus n'est prise en compte qu'une seule fois, même si, par exemple, on dénombre plusieurs Notre-Dame de la Garde. De ce fait, le chiffre total reste inférieur à celui que devrait donner la somme des titulatures distinctes de chaque catégorie de monuments (18), les édicules anciens et récents ayant en commun deux titulatures identiques.

Tous les pourcentages sont calculés par rapport au nombre total de titulatures mariales pour chaque catégorie d'oratoires anciens et récents.

9 et 10. Variété des vocables Notre-Dame.

considérer sous un nouveau jour l'appauvrissement du panthéon observé dans la catégorie des oratoires récents. Sans remettre en cause celui-ci, il semble cependant trouver une compensation dans une plus grande variété des vocables dédiés à la Vierge.

Si le répertoire des noms de saints s'affaiblit incontestablement, celui des noms de la Vierge paraît s'accroître en conséquence. Pour preuve : si l'on ajoute les titulatures distinctes des saints aux titulaires mariales distinctes (tableau $n^{\circ} 11$ ), l'écart s'élargit entre la catégorie des oratoires anciens et celle des monuments récents, au bénéfice de ces derniers : $59 \%$ de leurs dédicaces sont distinctes contre $46 \%$ seulement pour les oratoires anciens. Au vu de ces résultats, tout porte à croire que l'essor du culte marial masque en réalité la permanence d'une dévotion marquée par la possibilité de faire appel à un grand nombre d'intercesseurs différents ${ }^{(36)}$.

(36) On peut citer, à titre d'argument, l'anecdote suivante. L'église de Rians possède deux statues de la Vierge, l'une représentant Notre-Dame de Lourdes, l'autre. Notre-Dame de Nazareth. Seule la dernière, patronne de l'église et objet d'une grande dévotion locale, est toujours fleurie. Une Rianssaise se plaignit un jour auprès du curé du bourg de ne plus trouver de place pour disposer un vase de fleurs qu'elle venait d'apporter. Au prêtre qui lui conseilla de le mettre au pied de Notre-Dame de Lourdes, cette personne répondit alors: «Oh non! Ce n'est pas la même Vierge! » Cette réponse révèle des attitudes religieuses parfois fort éloignées des espoirs mis par le clergé dans un développement du culte marial. Le prêtre de la paroisse en question, consterné par la gentilité de ses ouailles, qualifie d'ailleurs la réflexion de la villageoise de "désespérante »... Dans le même registre, on peut se reporter à Gabriel LE BRAS, L'Eglise et le village, Paris, Flammarion, 1976, p. 266 ou à Ernest RENAN, Souvenirs d'enfance et de jeunesse, Paris, Calman-Lévy, 1967, pp. 19-20. 


\begin{tabular}{|l|c|c|}
\hline $\begin{array}{l}\text { Périodisation } \\
\text { des oratoires }\end{array}$ & Dédicaces connues & Dédicaces distinctes \\
\hline Anciens & 107 & $49 \quad(46 \%)$ \\
Récents & 64 & $38 \quad(59 \%)$ \\
Total & 171 & $69(40 \%)$ \\
\hline
\end{tabular}

11. Somme des titulatures distinctes des saints et des Notre-Dame (le total des dédicaces distinctes est inférieur à la somme des dédicaces distinctes de chaque catégorie pour la raison invoquée en note du tableau $n^{\circ} 9$ ).

Deuxième constat: le culte marial se caractérise par une nette préférence pour les Vierges rassurantes ou «joyeuses» $(52 \%$ des dédicaces $)^{(37)}$. Le pathétisme n'est pas de mise ici : $4,5 \%$ seulement des vocables peuvent suggérer une telle sensibilité. S'offre ainsi le constat de l'altérité de nombreux oratoires comparés aux croix de mission ou à celles de la Restauration, érigées bien souvent à des fins d'expiation.

Des différences importantes se dessinent encore entre les édicules anciens et récents. Les premiers regroupent $32 \%$ des appellations rassurantes, les seconds en rassemblent plus du double $(68 \%)$. Nous avons relevé une progression aussi nette dans la classe des dédicaces se référant à la Nativité, ce qui ne surprend pas puisqu'elle est un sousensemble de celle des Vierges rassurantes.

Ceci tend à nous conforter dans l'idée que la poussée mariale dans la catégorie des oratoires récents représente une compensation au recul des saints thaumaturges. Les fondateurs de ces édicules se tournent désormais vers une Vierge aux multiples visages, désignée explicitement comme apaisante, maternelle, accueillante, protectrice, capable de les écouter et de les rassurer aussi bien que les anciens intercesseurs.

\section{LE CHOIX DU NOM DU SAINT}

Parmi les différents intercesseurs auxquels se vouent les fondateurs d'oratoires, le choix d'un titulaire se fait pour des raisons variées : élection du saint tutélaire de la famille, adoption du saint honoré par une confrérie (saint Eloi ${ }^{(38)}$, sainte Barbe, saint Etienne) ou du nom du patron du village, du hameau ou de l'église. Dans les deux cantons, sur vingt-deux saints patrons de village, de hameaux ou d'églises, onze sont titulaires d'au moins un oratoire sur le finage. Ce sont, par exemple, sainte Foy et saint

(37) Ne sont pas pris en compte les vocables difficilement classables dans une catégorie ou dans une autre: les nombreuses Notre-Dame de I.ourdes - bien que le pouvoir miraculeux qui leur est attribué nous autoriserait à les ranger au sein des Vierges rassurantes - et certaines Notre-Dame pour lesquelles l'enquête de terrain n'a pas permis de lever la généralité de la titulature.

(38) Sur les sept oratoires dédiés à saint Eloi dans les deux cantons, deux sont érigés dans la commune du Beausset, où la Confrérie vouée à ce saint fait preuve d'un grand dynamisme, et quatre autres s'élèvent sur la commune de Saint-Julien le Montagnier où la fête de ce saint patron était autrefois célébrée avec ferveur par les villageois. 
Pierre à Artigues, respectivement patronne du village et patron de l'église; saint Eutrope et saint Alban au Beausset, premier et second patrons du bourg; saint Clair au Castellet, patron du village ou encore, dans le hameau des Rouvières, à Saint-Julien-le-Montagnier, sainte Philomène, patronne de la localité( ${ }^{39)}$.

On choisit aussi le saint patron d'une profession exercée par un des conjoints ou, tout simplement, un intercesseur dont un membre de la parenté, vivant ou décédé, porte le prénom. Le patronyme peut même être à l'origine du choix du titulaire: "C'est un saint Etienne parce que les propriétaires étaient des Etienne."

L'élection de la Vierge se fonde communément sur une forte dévotion mariale au sein de la famille, née du sentiment d'avoir reçu un message lors de la visite d'un sanctuaire qui lui est dédié, ou bien encore de la croyance en sa plus grande efficacité.

Ce dernier point pose inévitablement le problème de la puissance et de la popularité des saints : ces critères sont-ils déterminants dans le choix de tel ou tel vocable? Tous les intercesseurs n'ont pas le même prestige. A Rians, "saint Martin, il vaut rien. Il vaudrait mieux une sainte Vierge ", nous dira un villageois en désignant l'oratoire saint Martin. On trouve de «bons » saints et d'autres qui le sont moins, comme il y des Notre-Dame plus efficaces que d'autres ${ }^{(40)}$.

Si le choix du titulaire dépend sans doute du pouvoir que lui prêtent les autochtones, il n'existe pourtant pas un classement bien défini des intercesseurs en fonction de leur force respective, celle-ci restant de surcroît difficile à évaluer. Saint Martin qui «ne vaut rien » à Rians pourra être considéré comme un excellent intercesseur dans une autre commune varoise. L'appréciation de la force du saint dépend en fait de la tradition locale et familiale ${ }^{(41)}$.

Certains avancent « l'argument onomastique » pour expliquer le choix d'un ticulaire, en particulier dans le cas des saints thérapeutes ${ }^{(042}$. On solliciterait saint Clair pour soigner les maladies des yeux, ou saint Eutrope pour traiter l'hydropisie parce que la mise en relation du saint et

(39) Cela n'est pas systématique. A Signes, par exemple, l'église paroissiale est placée sous le rocable de saint Pierre-és-liens, sans qu'il y ait d'oratoire dédié à ce saint. A La Cadière, aucun monument n'a pour titulaire saint André, patron du lieu et de l'église. Il en va de même à Ginasservis et à Rians.

(40) Il suffit, pour s'en convaincre, de comparer des pèlerinages d'audience régionale comme celti de Notre-Dame des Grâces à Cotignac à ceux qui ont un rayonnement plus restreint (Notre-Dame du Beausset-Vieux par exemple).

(41) La force du saint procède également de la localisation de l'oratoire. La puissance du titulaire s'exercera d'autant mieux que l'emplacement choisi pour ériger le monument sera ce que Alban Bensa appelle une «maîtresse-place». Alban BENSA, Les saints guérisseurs du Perche-Gouët. Espace symbolique du Bocage, Paris Institut d'Ethnologie, 1978, p. 184). Là, le saint est plus fort qu'ailleurs. Ainsi, le saint Jean-Baptiste de SaintCyr, campé au sommet d'une colline dominant toute la région, est plus à même d'exercer son pouvoir titulaire que le saint Jean-Baptiste de la Cadière, situé dans lá plaine.

(42) Voir par exemple Marc BlOCH, Les rois thaumaturges, Paris, Gallimard, 1983, pp. 266267 : saint Marcoul avait la réputation de guérir les tumeurs scrofuleuses qui se logent de préférence au cou. Consulter également Eugène WEBER, La fin des terroirs, Paris, Fayard, Ed. Recherches, 1983, p. 501 et, plus généralement, toute la littérature des folkloristes sur le sujet. 
de la maladie résulterait d'une analogie verbale. Van Gennep a montré qu'un tel argument n'était recevable que pour quelques saints locaux, sans qu'on puisse jamais en faire une règle hagiographique ${ }^{(43)}$.

Le domaine dans lequel le saint exerce son pouvoir entre toutefois en considération, mais plus particulièrement pour les oratoires les plus anciens. Le choix relativement fréquent de saint Marc trouve probablement son origine dans son rôle privilégié au moment des Rogations ${ }^{(44)}$.

Les titulatures d'une partie des édicules illustrent également le souci de se protéger contre la maladie. En Provence, la «mort noire » ${ }^{(45)}$ et le choléra ${ }^{(46)}$ ont à maintes reprises provoqué de véritables paniques, et la dévotion aux saints anti-pesteux (saint Roch, saint Sébastien, saint Charles Borromée) et, plus généralement, aux saints thérapeutes, est à la mesure des dangers que représentaient ces fléaux et de l'angoisse qu'ils suscitaient.

Notons, pour finir, que les personnes qui prennent linitiative d'ériger un oratoire ne manifestent pas toujours de préférences particulières: "Nous avons mis la Vierge et Bernadette parce que nous les avions disponibles chez nous "; "J'ai mis saint Etienne parce que j'avais une statuette du saint. "De tels comportements contribuent probablement à la diffusion du culte marial: on dispose en effet plus facilement d'une statue de la Vierge que de celle d'un saint particulier.

La tolérance peut, dans ce domaine, s'avérer très grande : il n'y a pas toujours adéquation entre le vocable sous lequel est placé officiellement le monument et le saint représenté en réalité. A Rians comme au Beausset, des oratoires dédiés à Notre-Dame, Notre-Dame de la Garde, saint Louis de Gonzague, saint Joseph ou Notre-Dame de Lourdes abritent respectivement des statuettes de sainte Thérèse de l'Enfant Jésus, de Notre-Dame de Lourdes, de la Vierge à l'Enfant, du Sacré-Coeur ou de saint Joseph. Tout ceci dévoile un culte apparemment exempt de rigidités doctrinaires, laissant le champ libre à l'expression des sensibilités individuelles, voire aux déviations profanes.

(43) Arnold VAn GenneP, Manuel du folklore français contemporain, Paris, Picard, 19371958 , t. I, V, 3, p. 2530.

(44) "Les Rogations, c'est saint Marc, c'est le saint des Rogations », nous a déclaré une habitante de Rians en décrivant l'oratoire Saint-Marc. A Saint-Julien-le-Montagnier, où s'élève également un oratoire Saint-Marc, on ne procédait pas moins qu'à quinze bénédictions du terroir durant les trois journées de célébrations (Coutumier pour la paroisse de Saint-Julien en 1884, Archives diocésaines de Fréjus-Toulon).

(45) Au plus fort de l'épidémie de 1720, introduite par le navire le Grand Saint Antoine venu de Syrie, Monseigneur Belsunce, évêque de Marseille, prie régulièrement devant les oratoires érigés le long du chemir. qui conduit à Notre-Dame de la Garde. Nombreux sont les édicules qui commémorent cette période et rappellent la protection des saints ou de la Vierge contre un mal qui ranima les Confréries vouées à saint Roch et saint Sébastien. On compte vingt-sept oratoires dédiés aux saints anti-pesteux dans le Var, anciens pour la plupart.

(46) Des monuments furent érigés pendant l'épidémie de choléra morbus qui, en 1835 , fit plus de 3500 victimes dans le Var, surtout dans la région toulonnaise. (Michel MARgUERITTE, Histoire des Varois, Nice, Editions Sika, 1977, p. 68). Le XIX siècle a alors connu une renaissance triomphale du culte de saint Roch, quelque peu mis à mal au cours de la période révolutionnaire. 


\section{PRIVATISATION ET DÉVIATION PROFANE}

La privatisation est d'abord perceptible dans les changements qui affectent le choix des titulatures. On a pu en effet déceler deux modes différents d'allocation des noms de saints : celui des oratoires anciens, à vocation publique; celui des édicules récents, à vocation privée.

Deux grandes catégories de vocables, par conséquent, la première regroupant les noms des saints patrons de confréries et de corporations, de villages, de hameaux, ou d'églises, ou encore des hagiotoponymes tels que les noms de saints donnés à un quartier. Cette catégorie s'oppose à celle qui réunit les titulatures domestiques dont le nom, d'une part, ne peut être mis en relation avec aucune activité pratique ou symbolique de la communauté (fêtes, cérémonies, ordonnance toponymique du finage) et, d'autre part, honore un membre vivant ou décédé de la famille des fondateurs. Ces deux conditions sont nécessaires pour qu'un vocable soit considéré comme une dédicace privée.

A la lumière d'une telle distinction, on obtient des chiffres éloquents. Les oratoires anciens ont six fois plus de titulatures à vocation publique que les monuments récents. Inversement, les édicules récents ont 5,5 fois plus que les oratoires anciens des vocables à vocation privée ${ }^{(47)}$.

\begin{tabular}{|l|c|c|}
\hline $\begin{array}{l}\text { Périodisation } \\
\text { des oratoires }\end{array}$ & Vocables publics & Vocables domestiques \\
\hline Anciens & 41 & 4 \\
Récents & 7 & 22 \\
\hline
\end{tabular}

12. Vocables publics et domestiques.

Ces dernières données s'exposent à la critique car des entretiens avec tous les fondateurs d'oratoires anciens, si un tel travail était possible, conduiraient probablement à relever la part des vocables domestiques dans cette catégorie de monuments ${ }^{(48)}$. Néanmoins, pour incomplet qu'il soit, cet essai de quantification converge avec d'autres observations telles que la localisation préférentielle des oratoires récents dans l'enceinte de

(47) On retrouve l'opposition public/privé à l'intérieur même du groupe des titulaires patrons de corps de métiers : ceux qui sont titulaires d'oratoires récents s'opposent à ceux qui sont titulaires de monuments anciens. Ici, le corps de métier concerne une part jadis importante de la population active (saint Eloi pour les cultivateurs par exemple); là, c'est une personne privée exerçant un métier particulier (avocat par exemple), qui décide d'ériger un oratoire dédié au saint patron de sa profession (saint Yves au Beausset).

(48) Toutefois, en 1936, un auteur note l'extrême rareté des oratoires marqués d'un nom de famille (G. ARNAUD D'AGNiI, Léopold DOR, op. cit., p. 51). L'usage d'invoquer plus particulièrement son saint patron est donc peut-être un phénomène récent. Dans son étude des testaments avignonnais à la fin du Moyen Age, Jacques Chiffoleau observe que "dans bien des cas, le saint protecteur n'est pas celui dont on porte le prénom » (Jacques CHIFFoleaU, op. cit., p. 379). D'après Noël Coutet, à la même époque, dans le diocèse d'Aix, " la faveur des fidèles va rarement vers le saint dont ils portent le nom, mais plutôt vers des intercesseurs efficaces " (Noël COUIIIT, in Jean-Rémy PALANQUi:, Le diocèse d'Aix-en-Provence, Paris, Beauchesne, 1975, p. 72). 
propriétés privées, contrairement aux monuments anciens, presque toujours situés dans un espace public(19).

Tout cela révèle une tendance à la privatisation du culte: l'oratoire devient l'objet d'une dévotion privée (individuelle ou familiale) alors qu'il était jadis support d'une religiosité collective(50). Le passage des saints à vocation publique aux saints domestiques a pour corollaire la disparition des demandes collectives, ceci au bénéfice des requêtes privées. Naguère, les prières adressées aux saints concernaient des problèmes d'intérêt public: protection des récoltes ou arrêt d'une épidémie par exemple. Aujourd'hui, les demandes d'intercession sont centrées sur le groupe familial ou sur l'individu. Il s'agit désormais d'obtenir du saint son soutien pour telle ou telle affaire privée: maladie d'un membre de la famille, sauvegarde du patrimoine foncier, voyage, voire réussite au baccalauréat. Certes, ce type de rapport avec l'intercesseur représentait aussi une large part de la dévotion aux titulaires des oratoires anciens. Les deux formes de dévotion ne s'excluent pas mutuellement et coexistaient jadis. C'est précisément la disparition de l'une d'entre elles qui caractérise le culte rendu aux saints abrités dans les monuments récents ${ }^{(51)}$.

Par ailleurs, il faut observer que les raisons données par les fondateurs pour expliquer le choix d'un titulaire n'épuisent ni ne recoupent toujours les arguments avancés pour justifier l'érection d'un oratoire. Dans ce dernier cas, les réponses de certains fondateurs annonçant sans ambages l'avoir édifié parce que "ça fait provençal" ou parce que "ça fait rustique " imposent l'idée d'une altération possible des intentions religieuses qui devraient normalement présider à la construction d'un monument. Il y a là le signe d'une évolution vers un usage profane d'un oratoire désormais désacralisé, ce que semble confirmer la fonctionnalisation et l'esthétisation manifestes des édicules récents ${ }^{(j 2)}$.

(49) Si l'on prend comme critère de catégorisation l'accessibilité des monuments, $90 \%$ des oratoires anciens sont publics contre $15 \%$ seulement des édicules récents. C'est dire que la tendance à la privatisation des oratoires récents se dessine très clairement.

(50) Sur les 59 oratoires processionnels que nous avons inventoriés dans les deux cantons (monuments qui ont été le but de processions régulières ou exceptionnelles ou qui jalonnent les sentiers conduisant aux chapelles rurales), 58 sont des édicules anciens.

(51) Le problème de la privatisation des oratoires mériterait de plus amples développements, en ce sens qu'il touche à une question plus générale, la privatisation des lieux de culte, sujet qui a agité l'Eglise, les propriétaires fonciers et la masse des fidèles depuis pratiquement les débuts de la chrétienté. Voir par exemple, Henri PI.AT\&i.I.E, « La paroisse et son curé jusqu'à la fin du XIII" siècle ", in L'encadrement religieux des fidèles au Moyen Age et jusqu'au concile de Trente, Paris, C.T.H.S., 1985, $109^{\circ}$ Congrès National des Sociétés Savantes, Dijon, 1984, pp. 11-26.

(52) On relève d'une part le rajout d'objets fonctionnels sur ces édicules (boîte aux lettres, lanterne, robinet d'eau, portail - et, dans ce cas l'oratoire fait fonction de pilier d'entrée) et, d'autre part, une recherche esthétique (pierres apparentes parfaitement agencées, grille en fer forgé, utilisation de matériaux nobles: granit d'Allemagne, porphyre bleu du Dramont, pierre de Rognes) qui tranche avec l'architecture rudimentaire des monuments anciens. Ln outre, les oratoires récents ont en moyenne deux fois moins d'attributs religieux (croix, bénitier, agenouilloir) que les édicules anciens. Désormais semble primer le monument qui, toujours très travaillé sur le plan architectural, allie souci esthétique et intérêt fonctionnel. Pour de nombreux fondateurs désireux d'intégrer l'oratoire à leur conception du paysage régional, le monument doit inévitablement adopter le style néo-provençal si caractéristique des résidences secondaires. Dès lors, la croix, le bénitier et l'agenouilloir sont devenus superflus. 


\section{DE NOUVELLES PERSPECTIVES}

La privatisation de titulatures associées désormais à des monuments qui doivent être aussi des objets esthétiques suggère de nouvelles hypothèses. $\mathrm{Ne}$ faut-il pas voir dans cette évolution l'apparition chez le fondateur ou, tout au moins, le renforcement - d'une relation narcissique avec le monument et son titulaire?

En premier lieu, alors que naguère la mobilisation des intercesseurs se faisait avant tout pour signifier une identité collective, mobilisation dont on observait l'expression dans l'arrêt des processions des Rogations aux limites du finage ${ }^{(53)}$ et la prédominance des titulatures publiques, les oratoires récents semblent aujourd'hui renvoyer essentiellement à une identité individuelle ou à celle du groupe familial : en est-il un meilleur exemple que l'oratoire Notre-Dame de la Maintenance (supra p. 75) avec lequel le fondateur a voulu marquer la possession pluriséculaire d'une partie du terroir - c'est-à-dire marquer symboliquement un territoire par sa famille.

L'individualisation de chaque Notre-Dame et la privatisation des titulatures pourraient bien dénoter un désir d'appropriation de l'intercesseur, révélateur lui-même du souci d'affirmer une identité familiale ou individuelle, en rapport avec le territoire ${ }^{(54)}$. Le choix préférentiel du prénom des parents ou des grands-parents lors de l'allocation d'un nom de saint à un édicule récent répond très logiquement à sa localisation fréquente à l'entrée de la propriété, tel un blason, un armorial, délimitant avec plus ou moins d'ostentation le patrimoine familial ${ }^{(55)}$.

Si le titulaire a un rapport étroit avec une quête identitaire familiale ou individuelle ${ }^{(56)}$ - ce qui était probablement aussi le cas des oratoires

(53) A. VAN GENNEP, Manuel, op. cit., t. I, IV, 2, p. 1641. Alain Guerreau fait une observation identique dans le Mâconnais: «Les processions individualisaient radicalement les paroisses en les délimitant rituellement ». Alain GUERREAU, « Les pèlerinages du Mâconnais. Une structure d'organisation symbolique de l'espace », Ethnologie française, janvier-mars 1982, p. 16. Lors d'une procession un saint peut même refuser de franchir les limites de la paroisse en pesant plus lourdement que d'ordinaire sur les épaules de ses porteurs. Alban BENSA, op. cit., p. 182.

(54) Avec les titulatures Notre-Dame des Restanques, de la Maintenance, de la Bastide, etc., on est en présence d'appellations fondamentalement différentes des titulatures mariales génériques ou même des vocables Notre-Dame de la Garde ou de Lourdes qui désignent une Vierge a priori libre des personnes ou des groupes privés.

(55) Cela est particulièrement évident lorsque, comme cela se produit souvent avec les édicules récents, le nom de la propriété ou de la villa est inscrit sur le pilier de l'oratoire. Alors quautrefois se déplacer sur la commune c'étaic "passer simplement de la mouvance de saint Pierre à celle de saint Julien, ou de celle de saint Joseph à celle de Sainte Anne» (Lucienne A. RoUBIN, "Ordonnance toponymique en montagne provençale ", L'bomme bier et aujourd'bui. Recueil d'études en hommage à LeroiGourban, Paris, Cujas, 1973, p. 472), aujourd'hui les titulatures ne marquent plus l'espace communautaire mais bornent simplement des territoires privés. Et, effectivement, les édicules récents ne participent plus à l'ordonnance toponymique du terroir : sur 19 monuments dont la dédicace correspond directement à un toponyme communal (dénomination d'un chemin ou d'un quartier), un seul appartient au groupe des oratoires récents.

(56) Le fondateur d'un oratoire récent proclamera: "Cet oratoire, c'est l'bistoire de ma vie». 
anciens $^{(57)}$ - ce rapport semble désormais s'établir hors de toute référence à une identité collective. Même les déclarations de ceux qui érigent un édicule parce que " ça fait provençal " - et, dans le cas des nouveaux arrivants ${ }^{(58)}$, sans doute faut-il comprendre: "ça nous fait provençal" - ne doivent pas faire illusion ${ }^{(59)}$. Il s'agit moins de la manifestation d'une identité solidement ancrée dans les cadres traditionnels de la société provençale que de l'expression d'une autochtonie imaginaire s'accrochant, pourrait-on dire, aux «talismans d'un passé réinventé » ${ }^{(60)}$.

Anciennes ou récentes, les titulatures d'oratoires opèrent toutes comme classificateurs identitaires, mais à des niveaux différents: collectif ou privé. D'une certaine manière, et pour user d'une métaphore linguistique $^{(61)}$, ces titulatures voient aujourd'hui leur domaine de validité réduit à l'espace privé alors que jadis celui-ci s'étendait sur l'ensemble du terroir communal, et même au-delà ${ }^{(62)}$.

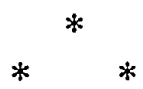

(57) Sur le rapport entre culte des saints et quête identitaire dans l'antiquité tardive, voir Peter BROWN, Le culte des saints. Son essor et sa fonction dans la chrétienté latine, Paris, Cerf, 1984, plus particulièrement le chapitre « Le compagnon invisible », pp. 71 93.

(58) Alors que $84 \%$ des édicules anciens sont dus à l'initiative d'agriculteurs, $67 \%$ des monuments récents sont érigés par des non-agriculteurs. Parmi ces derniers, nombreux sont ceux qui ont contribué à l'essor démographique considérable connu par la région ces trois dernières décennies : la population des deux cantons a fait plus que doubler entre 1954 et 1982.

(59) Il y a une part d'artificiel à vouloir opposer, comme nous le faisons, identité collective et identité individuelle: toutes deux sont en réalité inséparables. L'appartenance au groupe constitue elle-même, pour le sujet individuel, un élément de sa propre identité, et les fondateurs qui déclarent avoir érigé un oratoire parce que " ça fait provençal " ne s'y trompent pas puisqu'en exprimant ainsi leur appartenance à une région, ils affirment leur propre identité. Ce qui nous paraît changer avec les titulatures récentes, c'est que l'image du moi à laquelle elles renvoient prévaut désormais sur celle du groupe d'appartenance.

(60) J. LE GOFF, cité par Joël SAUGNIEUX, «Elitisme et cléricalisme dans l'Eglise espagnole des Lumières: le problème de la "potestas praedicandi" ", in La religion populaire, Paris, C.N.R.S., 1979, p. 249.

(61) Voir à ce sujet: Emile BENVEnisTe, Problèmes de linguistique générale, Paris, Gallimard, 1974, t. II, p. 52.

(62) On songe ici aux rapports existant entre « orgueil du clocher » et culte des saints (voir P. SAINTYVES, En marge de la Légende dorée, Paris, Ed. Emile Nourry, 1930, pp. 248 255). Chaque commune possède un certain nombre de titulaires, présents nulle part ailleurs, et qui de ce fait la distinguent des autres localités : sur les 136 communes varoises dotées d'oratoires, 57 saints sont cités une seule fois, soit $41 \%$ au total des dédicaces distinctes, et 13 intercesseurs sont invoqués deux fois seulement. On peut supposer que le particularisme de clocher a cessé de jouer à partir du moment où les monuments privatisés, ne pouvant plus être vus et reconnus parce que désormais cachés et méconnus, ont perdu toute vale:ar symbolique pour les autochtones autres que les fondateurs. 
Ces réflexions n'ont d'autre ambition qu'explorer une voie de recherche sur laquelle nous souhaitons nous engager : celle des relations entre culte des saints et quête identitaire ${ }^{(63)}$. Nous voilà loin, en tout cas, de nos préoccupations initiales. Il est vrai qu'on ne doit jamais préjuger de la richesse d'un objet ethnographique: pouvait-on en effet supposer que l'étude d'un monument apparemment banal débouche sur ce type de spéculations? Van Gennep l'avait pourtant pressenti, lui qui voyait dans l'oratoire "une image pieuse composite » qui, sans cesse, a changé d'apparence au cours des dix derniers siècles pour s'adapter aux besoins locaux ${ }^{(64)}$. L'enquête menée sur les édicules varois en apporte, croyonsnous, le témoignage : un même monument peut être doté de fonctions et de sens différents ${ }^{(65)}$ selon les lieux et les époques.

Joël Candau

Saint-Laurent-du-Var

(63) Thème que nous avons développé dans «Oratoires varois et quête identitaire: le calendrier et le territoire ", communication présentée au colloque de la Société d'Ethnologie française: Etbnologie des faits religieux en Europe, Strasbourg, 24, 25 et 26 novembre 1988 (actes à paraître).

(64) A. VAN GENNEP, "Patronages, chapelles et oratoires de la Haute Maurienne. Etude statistique et critique », Revue d'Histoire de l'Fglise de France, tome XXV, p. 182. II faut par conséquent souligner les limites des entreprises consistant à vouloir appréhender dans la synchronie des objets historiquement hétérogènes, labeur favori de certains folkloristes: l'erreur de nombreux ouvrages sur les oratoires consiste à uniformiser, amalgamer, des monuments d'âges divers, érigés dans des régions souvent fort dissemblables et, de ce fait, pourvus de sens probablement fort différents par les autochtones.

(65) Notons au passage que la variabilité historique de la signification des oratoires nous rappelle opportunément qu'il est dans la nature des faits symboliques de navoir jamais que :e sens qui leur est assigné par l'exégèse à laquelle se livrent continuellement les individus et les groupes. A ce propos, consulter Dan SPERBER, Le symbolisme en général, Paris, Hermann, 1974, bibliogr., 163 p. 